\title{
Fabrication of zinc oxide nanoneedles on conductive textile for harvesting piezoelectric potential
}

\author{
Azam Khan, Mushtaque Hussain, Omer Nur and Magnus Willander
}

\section{Linköping University Post Print}

\section{Tweet}

N.B.: When citing this work, cite the original article.

Original Publication:

Azam Khan, Mushtaque Hussain, Omer Nur and Magnus Willander, Fabrication of zinc oxide nanoneedles on conductive textile for harvesting piezoelectric potential, 2014, Chemical Physics Letters, (612), 62-67.

http://dx.doi.org/10.1016/j.cplett.2014.08.009

Copyright: Elsevier

http://www.elsevier.com/

Postprint available at: Linköping University Electronic Press

http://urn.kb.se/resolve?urn=urn:nbn:se:liu:diva-111603 


\title{
Fabrication of zinc oxide nanoneedles on conductive textile for harvesting piezoelectric potential
}

\author{
Azam Khan*, Mushtaque Hussain, Omer Nur and Magnus Willander \\ Department of Science and Technology (ITN), Linköping University, Campus Norrköping, 60 \\ 174 Norrköping, Sweden
}

Corresponding Author: Azam Khan

Email: azam.khan@liu.se

\begin{abstract}
Keeping the fact in mind that different morphologies have strong influence on piezoelectric properties, $\mathrm{ZnO}$ NNs were synthesized on textile for harvesting piezoelectricity. Piezoelectric potential was captured from $\mathrm{ZnO}$ NNs grown on textile by using AFM in contact mode. Structural study was carried out by using FESEM, HRTEM and XRD techniques. The recorded output potential and current was more than $45 \mathrm{mV}$ and $150 \mathrm{nA}$. The combination of $\mathrm{ZnO} \mathrm{NNs}$ and textile can be used effectively for energy harvesting applications and the use of textile fabric can pave the way for cheap, flexible, wearable, washable and environment friendly nanodevices.
\end{abstract}

\section{Keywords}

Conductive textile, $\mathrm{ZnO}$ nanoneedles, aqueous chemical growth method, piezoelectric potential

\section{Introduction}

In recent years the use of technology has been increased very rapidly worldwide as the population of the world. The development of society depends on the available amount of energy or electricity. Due to heavy consumption of energy in every field of life the available resources will not be sufficient in near future to fulfil the energy requirements [1]. Therefore, research 
community is trying to invent alternative and cost effective resources for generation of energy / electricity with several features like environment friendly, light weight, flexible, wearable, cheap and nontoxic with high power generation from small area [2-3].

Different techniques have been utilized for harvesting electricity such as electrostatic induction, photoelectric effect, thermoelectric effect, piezoelectric effect and electrochemical reactions etc [4-8]. In all these techniques, the phenomenon of self-power generation has been utilized extensively for harvesting piezoelectric potential, because it is simple, cost effective, and nonhazardous and has capability to produce larger amount of electricity.

The backbone of this technique is the use of piezoelectric materials such as $\mathrm{ZnO}, \mathrm{GaN}$, $\mathrm{CdS}$, and $\mathrm{ZnS}$. But due to high electromechanical coupling coefficient zinc oxide $(\mathrm{ZnO})$ was found to be more suitable candidate. Moreover, $\mathrm{ZnO}$ possess excellent properties such as large surface to volume ratio, high thermal and chemical stability, large exciton binding energy (60 $\mathrm{meV}$ ), high flexibility and largest piezoelectric coefficient [9]. Beside this $\mathrm{ZnO}$ probably holds the largest family of nanostructures like nanorods (NRs), nanowires (NWs), nanoflowers (NFs) and nanotubes (NTs), which have been utilized for harvesting piezoelectricity [2-3, 10-13]. Zhao et al. synthesized a new class of $\mathrm{ZnO}$ nanostructure as multipod, nanostructures of $\mathrm{ZnO}$ that exhibited excellent photoluminescence characteristics [14]. Another unique class of $\mathrm{ZnO}$ nanostructures called as multipod $\mathrm{ZnO}$ with nanonails was also used for analysis of optical properties [15]. Similarly a classical study was performed by synthesizing cage-like nanotetrapods of $\mathrm{ZnO}$ nanostructure for the investigation of optical characteristics [16]. It is important to point out that $\mathrm{ZnO}$ NNs based nanogenerator was first developed on silicon substrate by using high temperature thermal evaporation growth technique [17].But using textile as substrate no experimental report is available on low temperature growth of $\mathrm{ZnO}$ nanoneedles (NNs) for harvesting piezoelectric potential. Literature reveals that an extensive investigation has been reported regarding the piezoelectric properties of single crystalline $\mathrm{ZnO}$ NRs by using atomic force microscopic technique [2]. In another investigation Wang et al. have been investigated regarding the mechanical and piezoelectric properties of $\mathrm{ZnO} \mathrm{NWs}$ by using $\mathrm{AFM}$ technique [18]. Kim et al. have performed an extensive investigation regarding the piezoelectric properties of $\mathrm{ZnO}$ nanosheets/nanowalls [19]. Similarly Khan et al. have investigated the piezoelectric potential generation from $\mathrm{ZnO}$ nanoflowers (NFs) [3]. In another study, $\mathrm{Xi}$ et al. demonstrated the piezoelectric properties of ZnO NTs by using atomic force microscopy [20]. Experimental 
results on the lateral bending of piezoelectric nanostructures have been reported by Wang et al [21]. Beside these various theoretical investigations are also available on harvesting piezoelectricity from semiconducting materials. More importantly Falconi et al first explained the importance of different positions of contacts, types of mechanical input (e.g. vertical compression versus lateral bending), and morphology (e.g. nanowalls). They presented the theory for harvesting piezoelectricity from ZnO NWs and nanowalls (NWLs) related to the other experimental results and designed a highly efficient piezoelectric nanogenerator [22]. The advantages of tapered piezoelectric nanostructures (under vertical compression) have been first recognized by Araneo et al. They demonstrate that, how $\mathrm{ZnO}$ nanostructures like n-type and ptype cylindrical and conical NWs can be used successfully for harvesting piezoelectricity. Another theoretical calculation regarding the piezopotential in lateral bending of piezoelectric tapered nanostructures have been reported, which extensively discussed the possible crucial advantages [23]. Various studies have been published previously that, different morphologies of $\mathrm{ZnO}$ nanostructures have strong influence on their piezoelectric properties as shown in table 1.

In this study, $\mathrm{ZnO} \mathrm{NNs}$ were grown on conductive textile fabric substrate by using aqueous chemical growth method. Structural analysis like shape, diameter, length, growth orientation and crystalline quality were performed by using FESEM, HRTEM and XRD techniques. The measurement for harvesting piezoelectric potential was examined by using AFM in contact mode.

\section{Experimental}

$\mathrm{ZnO} \mathrm{NNs}$ were grown on commercially available conductive textile fabric (ArgenMesh: Less EMF Inc. USA) substrate by using aqueous chemical growth method as shown in figure 1 (a). A typical aqueous chemical growth (ACG) method was used for the growth of ZnO NNs [24]. Aqueous solution was prepared by taking equimolar concentration of zinc acetate and hexamethylenetetramine and dissolved in $200 \mathrm{ml}$ deionized water by using magnetic stirrer. To change the shape of desired structure $8 \mathrm{ml}(25 \%)$ of ammonia solution was added to adjust the $\mathrm{pH}$ in to the growth solution. All experimental work for the growth of NNs was performed in cleanroom to avoid the contamination. Prior to the growth of $\mathrm{ZnO} \mathrm{NNs}$, textile fabric substrate was cleaned with acetone, isopropanol and deionized water respectively for 4-5 min by using electronic sonication bath. After cleaning the substrate a layer of $\mathrm{ZnO}$ nanoparticles containing 
seed solution was spin coated at $4000 \mathrm{rpm}$ for $30 \mathrm{sec}$. This process was repeated three times and then sample was heated on $100^{\circ} \mathrm{C}$ for several minutes to achieve a good adhesion of seed layer on the surface of the substrate. After that the sample was fixed in a Teflon sample holder and dipped in to the precursor solution. Then it was covered by aluminum foil and placed it into an oven at a temperature of $90^{\circ} \mathrm{C}$ for 6 hours. After this period oven was turn off and left the beaker in the oven to cool down naturally. Then grown $\mathrm{ZnO}$ NNs were washed by deionized water and dried by flow of nitrogen. Figure 1 (b) is showing the digital microscopic image of grown $\mathrm{ZnO}$ NNs on conductive textile fabric, while the schematic illustration of growth process is shown in figure 2 .

Field emission surface scanning electron microscopy (FESEM) of model (LEO-1550 Gemini) was used at $15 \mathrm{kV}$ for analysis of growth morphology of $\mathrm{ZnO}$ NNs. High resolution transmission electron microscopy (HRTEM) of model (FEI Tecnai G2 TF20 UT) was used at $200 \mathrm{kV}$ field emission gun with point resolution of $1.9 \AA$ for structural properties and X-ray diffraction (XRD) instrument (Phillips PW 1729) equipped with CuK $\alpha$ radiation $(\lambda=1.5418 \AA$ ) with a generator of voltage $40 \mathrm{kV} /$ current $40 \mathrm{~mA}$ was used for crystal analysis.

Atomic force microscopy (digital instrument in multimode, Netherlands) and custommade trans-impedance amplifier with platinum coated probes (NT-MDT NSG11/Pt, Russian Federation) were used for the measurement of piezoelectric potential generated from the $\mathrm{ZnO}$ NNs. All the measurements were performed in contact mode with fast scanning speed $30 \mu \mathrm{m} / \mathrm{s}$ and the scan direction was parallel to the cantilever. Initially cantilever deflection signal was set at zero with the cantilever un-deflected and the set point was set between 1-5 volts. The tip spring constant was typically $0.6 \mathrm{~N} / \mathrm{m}$ to maintain the applied force between 0.6 to $3 \mu \mathrm{N}$. The sensitivity of the deflection signal was $100 \mathrm{~nm} / \mathrm{V} \pm 20 \%$ and a separate sample holder was used for the piezoelectric current measurement. A gain of the transimpedance amplifier was $1 \mathrm{mV} / \mathrm{nA}$ from the feedback resistor. A positive (+) output voltage is indicating that the current is flowing from the tip into the sample in entire circuit. For controlling the leakage current, an operational amplifier having gain bandwidth product of around $1.8 \mathrm{MHz}$ was used to keep the sample at ground potential. This gain-bandwidth produced an effective impedance of $500 \Omega$ to the ground at $1 \mathrm{kHz}$ with the $1 \mathrm{M} \Omega$ feedback resistors. The input bias current of the amplifier was less than $25 \mathrm{pA}$ and no external voltage was applied at any stage of measurement [2-3]. 


\section{Results and discussion}

Figure 3 is revealing the field emission SEM images of grown $\mathrm{ZnO}$ NNs on conductive textile in low and high magnifications. Figure 3 (a) exhibits the homogeneous and high density growth of $\mathrm{ZnO} \mathrm{NNs}$ with well alignment in c-axis direction. The architecture of $\mathrm{ZnO}$ NNs is hexagonal wurtzite shape, the bottom diameter is around 450 - $500 \mathrm{~nm}$ and sharp top edges have diameter around $10-20 \mathrm{~nm}$ as shown in figure 3 (b). It was also seen that the all the grown NNs are almost equal in length. Structural study of grown NNs was also investigated by using high resolution TEM as shown in figure 4. Figure 4 (a) is displaying the image of NNs in low magnifications. Comparison of image with scale bar confirms that, the diameter of base of NNs is $450-500 \mathrm{~nm}$ and diameter of top is around $10-20 \mathrm{~nm}$. Figure 4 (b) is expressing the diffraction pattern of $\mathrm{ZnO}$ NNs. Also growth orientation $\mathrm{ZnO}$ NNs in c-axis direction associated to hexagonal wurtzite nanostructures can be clearly seen. In figure 4 (c), high magnification image is showing the crystalline quality of grown $\mathrm{ZnO} \mathrm{NNs}$ and the c-axis direction of lattice structure.

Figure 5 is the typical XRD of the grown $\mathrm{ZnO}$ NNs on silver coated conductive textile fabric substrate. The diffractions peaks (100), (002), (101) and (102) are reflected from the different planes of $\mathrm{ZnO}$ NNs and two more peaks are associated with silver film [2-3]. The domination of (002) peak indicated that, the grown $\mathrm{ZnO}$ NNs are vertically well aligned in c-axis direction with good crystalline quality.

Figure 6 (a) displays the model of grown $\mathrm{ZnO}$ NNs and a hexagonal top view of the $\mathrm{NN}$ architecture are also given in figure, which is expressing the little dark top of NNs along with bottom image. As shown in figure 6 (b) bottom electrode was grounded with Ag paste and Pt. coated AFM tip was used as Schottky contact [25-26]. When an external load was employed on ZnO NNs by AFM tip then due to deformation/ bending of NNs positive $\left(\mathrm{V}^{+}\right)$and negative $\left(\mathrm{V}^{-}\right)$ charges created along the stretched and compressed sides of $\mathrm{ZnO}$ NNs respectively. If tip is touching compressed or stretched sides of the NNs, then it will only cause forward or reverse bias Schottky junction [27-28]. Figure 6 (c) showing the top view of $\mathrm{ZnO} \mathrm{NN}$ model, where due to the deformation/ bending polarization has been occurred along the $\mathrm{ZnO} \mathrm{NN}$ [5].

The procedure of scanning by AFM was very fast therefore the demonstration of bending during the scanning has been explained by Figure 7. Different positions of AFM tip during the contact with $\mathrm{ZnO}$ NNs are revealing the stepwise process of piezoelectricity generation. Figure 7 
(a) is showing the position of AFM tip just before the start of scan measurements. When an external load was applied on ZnO NN by AFM tip then NN started to bend in the direction of applied force $\left(\mathrm{F}_{\mathrm{y}}\right)$ and no output potential was observed as shown in figure 7 (b). The piezoelectric potential was captured at maximum bending of NNs, at the maximum bending charges accumulates from edges of NNs and forms an electric field in the direction of applied load/ vertical force as shown in figure 7 (c). When tip released the NN then as soon as charges restored to zero and NN restored its original position as shown in figure 7 (d). The mechanism of piezoelectricity generation from $\mathrm{ZnO} \mathrm{NNs}$ is similar to the conical or cylindrical like nanostructures as define in published work [29]. As the diameter of top and bottom of NNs were different therefore free charge carriers and piezoelectric potential will vary due to the difference of strain / stress at bottom and top. Since the tip of NN will stretch more due to the inhomogeneous diameter, therefore the thin top will show better mechanical stability as compared to bottom. The amount of generated charges at the top will be more in magnitude as compared to bottom [29].

Figure 8 (a) is showing the topographic image of $\mathrm{ZnO}$ NNs during the scanning. The sharp edges (top) of NN are displaying the bending due the external force/ load applied during the scanning by AFM platinum (Pt.) coated tip. The output potential generation process depends on the Schottky junction formation between the semiconductor and the tip of AFM according to the fundamental theory of piezoelectricity [30]. The linear electromechanical coupling of the material is the phenomenon which converts the mechanical strain into ionic polarization of charges, these charges generates piezoelectric potential and modulated the electron transportation process in the NNs [31]. Figure 8 (b) demonstrates the current transport perturbation of grown $\mathrm{ZnO}$ NNs and the non-linearity of the curve indicates the Schottky junction formation between the grown $\mathrm{ZnO}$ NNs and the AFM Pt. coated tip. It can be also confirmed by the relation:

$$
\phi_{b}=\phi_{m}-\chi_{s}=\phi_{P t}-\chi_{Z n O}=6.1-4.3=1.8 \mathrm{eV}
$$

Where $\phi_{b}$ is the barrier height, $\phi_{m}$ is the work function of platinum and $\chi_{s}$ is the electron affinity of $\mathrm{ZnO}$ [27-28]; Figure 8 (c) is indicating that, the generated output piezoelectric potential from $\mathrm{ZnO}$ NNs was recorded in 3D. When AFM tip deflected (stretched) the ZnO NNs then polarization has been occurred due the activation of internal energy of the molecules. NNs lattices crystals start to accumulate positive charges from the stretched side and negative from the compressed side and form an electric field. This phenomenon of linear electromechanical 
coupling is taking into account by the AFM tip converts the mechanical energy into electrical energy. It was observed that, the piezoelectric potential was captured at the maximum bending before leaving the NNs by AFM tip and suddenly voltage reached at zero after releasing from the tip [27-31]. The maximum output potential was captured up to more than $45 \mathrm{mV}$. Figure 8 (d) is exposing the line profile of exerted current amount from $\mathrm{ZnO}$ NNs during the scanning by AFM tip corresponding to the resulting output potential. When AFM tip was scanning the over the vertical grown $\mathrm{ZnO}$ NNs then several peaks of generated current were recorded and the amount of piezoelectric current was more than $150 \mathrm{nA}[2-3]$.

\section{Conclusion}

Conductive textile fabric was used for the fabrication of $\mathrm{ZnO} \mathrm{NNs}$ based nanogenerator for harvesting piezoelectric potential. AFM in contact was used for measurement of generated output potential from $\mathrm{ZnO}$ NNs. The amount of piezoelectric potential was measured to be more than $45 \mathrm{mV}$ and the corresponding current was more than $150 \mu \mathrm{A}$. Nonlinearity of I-V curve demonstrates the good Schottky behaviour between AFM tip and ZnO NNs. The obtained results confirmed that different morphologies have strong influence on piezoelectric properties of $\mathrm{ZnO}$ nanostructures and NNs can be used as effectively as other morphologies. Moreover the use of textile as an alternative substrate for the fabrication of nanogenerators is also beneficial as the advantages of textile like cheap, non-toxic, washable, foldable, and wearable can be easily added in the architecture of nanogenerators.

Table 1: The amount of piezoelectric potential generated from different morphologies of $\mathrm{ZnO}$ nanostructures.

\begin{tabular}{|c|c|c|c|}
\hline S. No. & Nanostructures & Piezoelectric potentials & References \\
\hline 1 & Nanorods & $10 \mathrm{mV}$ & {$[2]$} \\
\hline 2 & Nanowires & $5-35 \mathrm{mV}$ & {$[11]$} \\
\hline 3 & Nanowalls & $400 \mathrm{mV}$ & {$[15]$} \\
\hline 4 & Nanoflowers & $600 \mathrm{mV}$ & {$[3]$} \\
\hline 5 & Nanotubes & $36 \mathrm{mV}$ & {$[16]$} \\
\hline 6 & Nanoneedles & More than $45 \mathrm{mV}$ & Present work \\
\hline
\end{tabular}




\section{References}

[1]. G. Zhu, J. Chen, T. Zhang, Q. Jing, Z. L. Wang, Nature Communications 2014, 5, 3426

[2]. A. Khan et al., Appl. Phys. Lett. 2012, 101, 193506

[3]. A. Khan, M. A. Abbasi, O. Nur, M. Willander, Phys. Statue Solidi (RRL) 2013, 7(11), 980

[4]. M. Gräzel et al., Nature 2001, 414, 338

[5]. Z. L. Wang, J. H. Song, Science 2006, 312, 242

[6]. D. Kraemer et al. Nat. Mater. 2011, 10, 532

[7]. M. K. Debe et al., Nature 2012, 486, 43

[8]. G. Zhu et al., Nano Lett. 2012, 12, 4960

[9]. R. Agrawal, H. D. Espinosa, Nano Lett. 2011, 11, 786

[10]. A. Khan, M. Hussain, O. Nur and M. Willander, J. Phys. D: Appl. Phys. 2014, 47

[11]. M. Riaz, J. H. Song, O. Nur, Z. L. Wang, M. Willander, Adv. Funct. Mater. 2010, 21, 628

[12]. S. Niu et al., Adv. Mater. 2013, 25(27), 3701

[13]. Y. Gao, Z. L. Wang, Nano Lett. 2007, 7, 2499

[14]. Y. N. Zhao, M. S. Cao and J. G. Li, J. Mater. Sci. 2006, 41 (8) 2243

[15]. Y. N. Zhao et al., Scripta Mater. 2006, 54 (12), 2057

[16]. Y. N. Zhao et al., J. Nanosci. Nanotechno. 2006, 6 (8), 2525

[17]. C. Periasamy and P. Chakrabarti, J. Appl. Phys. 2011, 109, 054306

[18]. X. Wang, J. H. Song, Z. L. Wang, J. Mater. Chem. 2007, 17, 711

[19]. K. H. Kim et al., Scientific Reports 2013, 3, 2017

[20]. Y. Xi et al., J. Mater. Chem., 2009, 19, 9260

[21]. X. Wang, J. H. Song, F. Zhang, C. He, Z. Hu and Z. L. Wang, Adv. Mater., 2010, 22, 2155

[22]. C. Falconi et al., Sensor Actuat B-Chem., 2009, 139, 511

[23]. R. Araneo and C. Falconi, Nanotechnology 2013, 24, 265707

[24]. A. Khan et al., J. Mater. Sci. 2014, 49, 3434

[25]. J. H. Song, X. Wang, J. Liu, H. Liu, Y. Li, Z. L. Wang, Nano Lett. 2008, 8, 203

[26]. J. H. Song, J. Zhou, Z. L. Wang, Nano Lett 2006, 6, 1656

[27]. Y. F. Lin, J. H. Song, Y. Ding, S. Y. Lu, Z. L. Wang, Adv. Mater. 2008, 20, 3127

[28]. Y. F. Hu, Y. Zhang, C. Xu, L. Lin, R. L. Snyder, Z. L. Wang, Nano Lett. 2011, 11, 2572

[29]. R. Araneo, G. Lovat, P. Burghignoli and C. Falconi, Adv. Mater., 2012, 24, 4719

[30]. Z. L. Wang et al., Adv. Funct. Mater. 2008, 18, 3553 
[31]. X. Wang, J. H. Song, J. Liu, Z. L. Wang, Science 2007, 316, 102

Figures with captions

Figure 1:

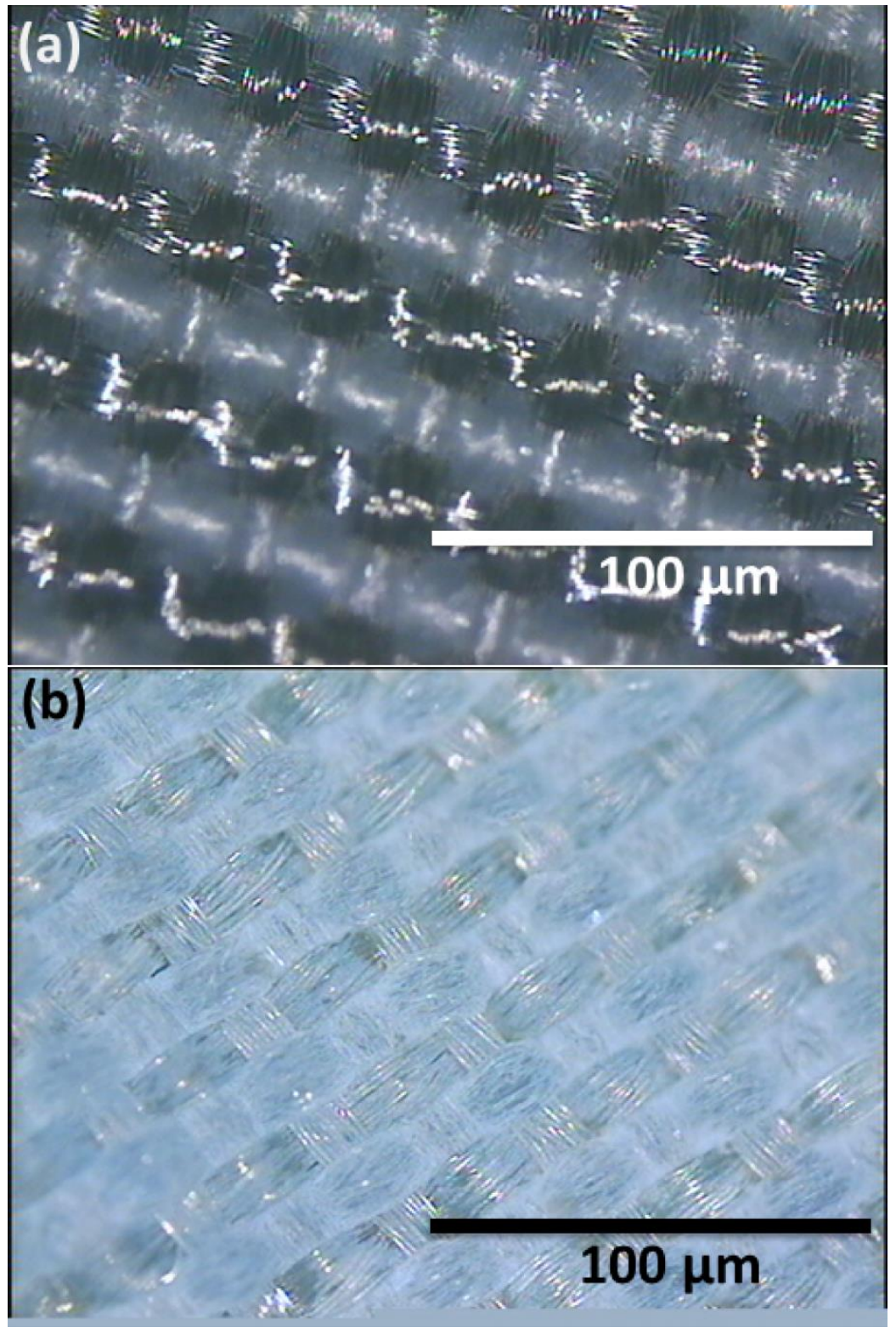

Figure 1: Micrographic images of ArgenMesh conductive textile (a) prior to the growth of $\mathrm{ZnO}$ NNs (b) after the growth of ZnO NNs. 
Figure 2:

(a) Seed layer deposition process

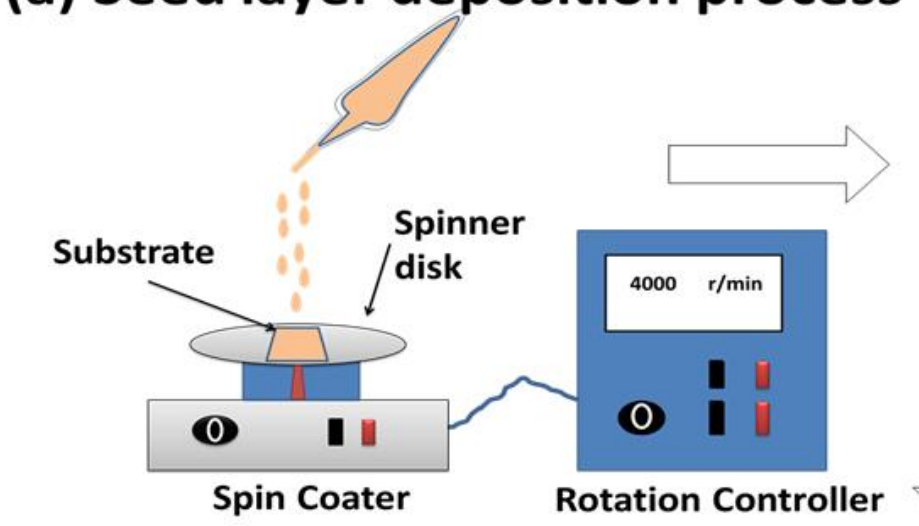

(b) Soft baking

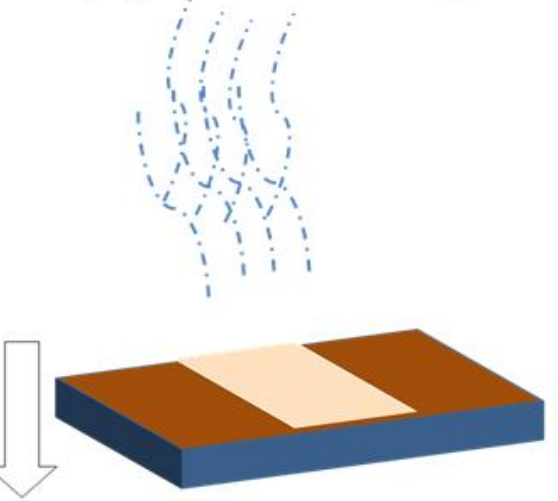

(d)

(c) Growth

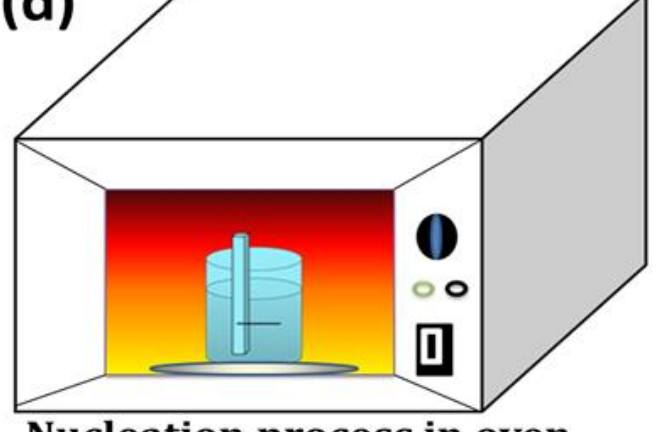

Nucleation process in oven at a temperature $90^{\circ} \mathrm{C}$. (c) Growth solution and

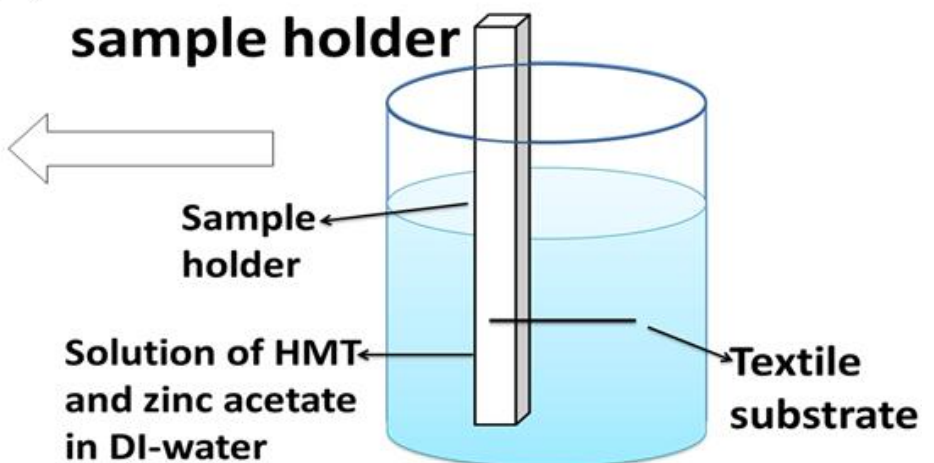

Figure 2: Schematic illustration of growth procedure for $\mathrm{ZnO}$ NNs. 
Figure 3:

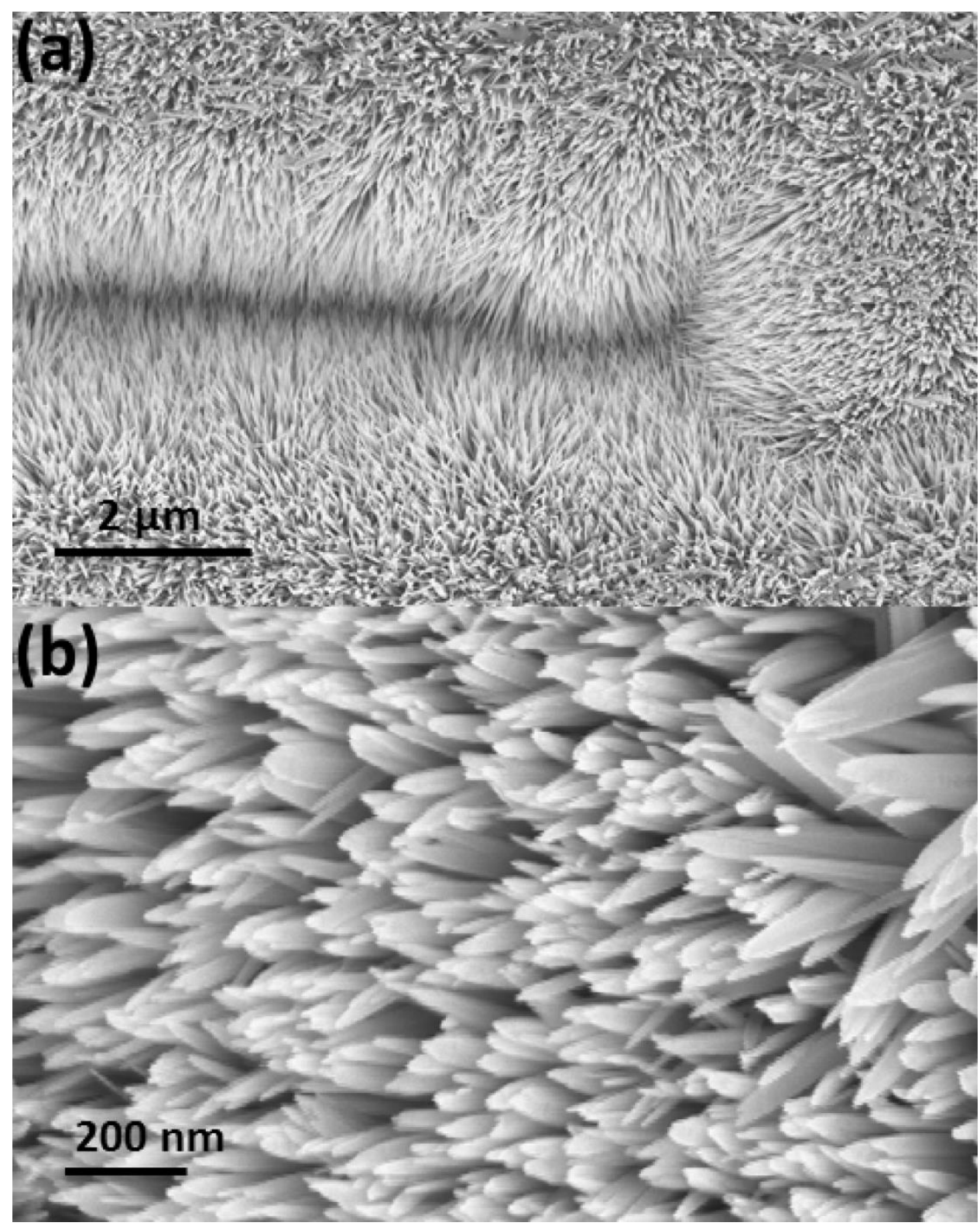

Figure 3: SEM images of grown $\mathrm{ZnO}$ nanoneedles (a) low resolution is image showing the growth density of nanoneedles (b) high resolution image clearly expressing the sharp top of grown nanoneedles. 
Figure 4:

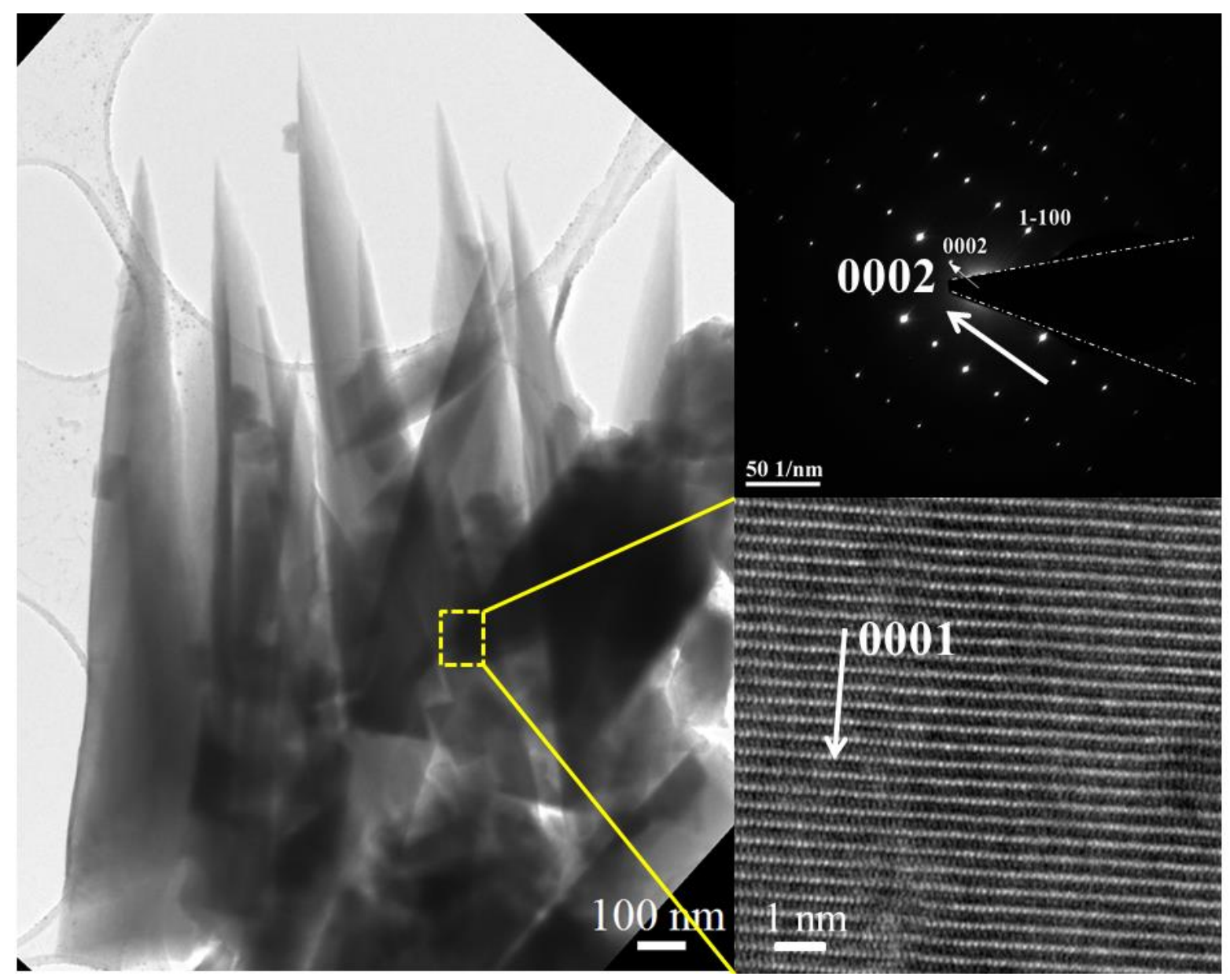

Figure 4: High resolution TEM images of $\mathrm{ZnO}$ NNs grown on textile (a) low magnification image, (b) diffraction pattern of $\mathrm{ZnO} \mathrm{NNs}$, (c) high magnification image of selected area. 
Figure 5:

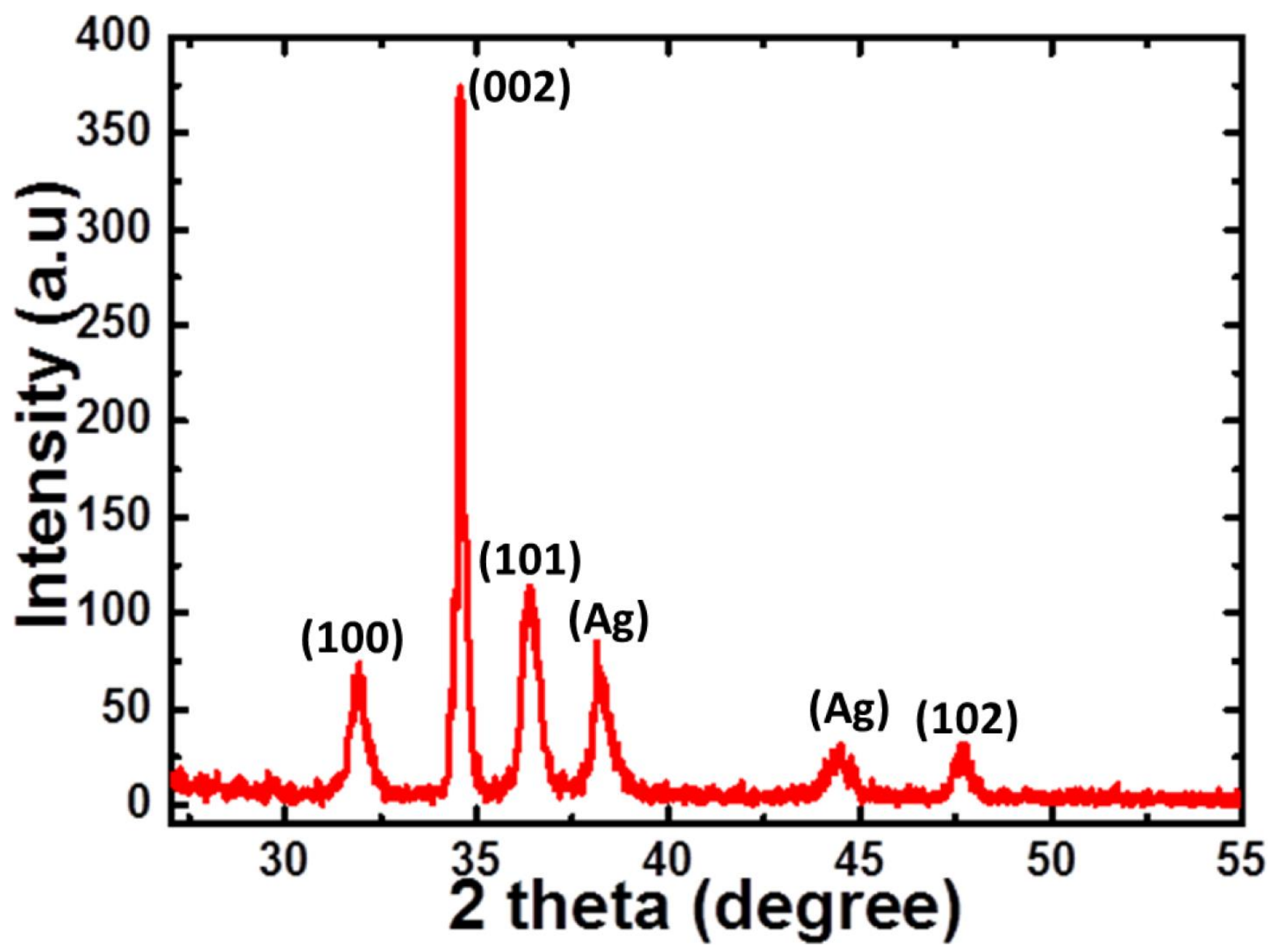

Figure 5: Typical XRD of grown $\mathrm{ZnO} \mathrm{NN}$ grown on (ArgenMesh) conductive textile fabric substrate. 
Figure 6:

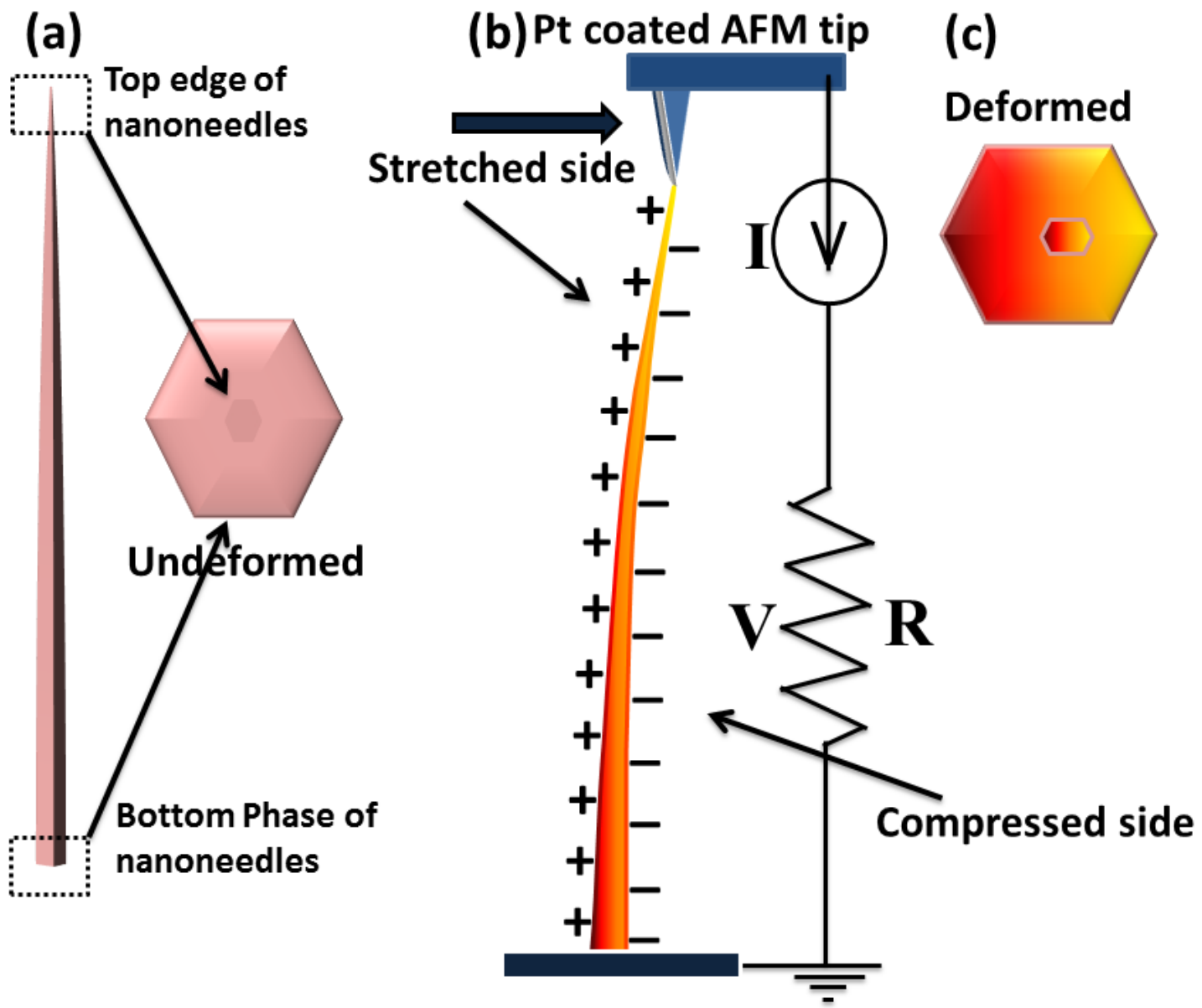

Figure 6: (a) A model showing the top and bottom hexagonal surfaces of ZnO NNs. (b) A model of AFM tip's scanning and piezoelectricity generation process from $\mathrm{ZnO} N \mathrm{~N}$. (c) Top view of $\mathrm{NN}$ induced polarization during deformation/bending. 
Figure 7:

(a)

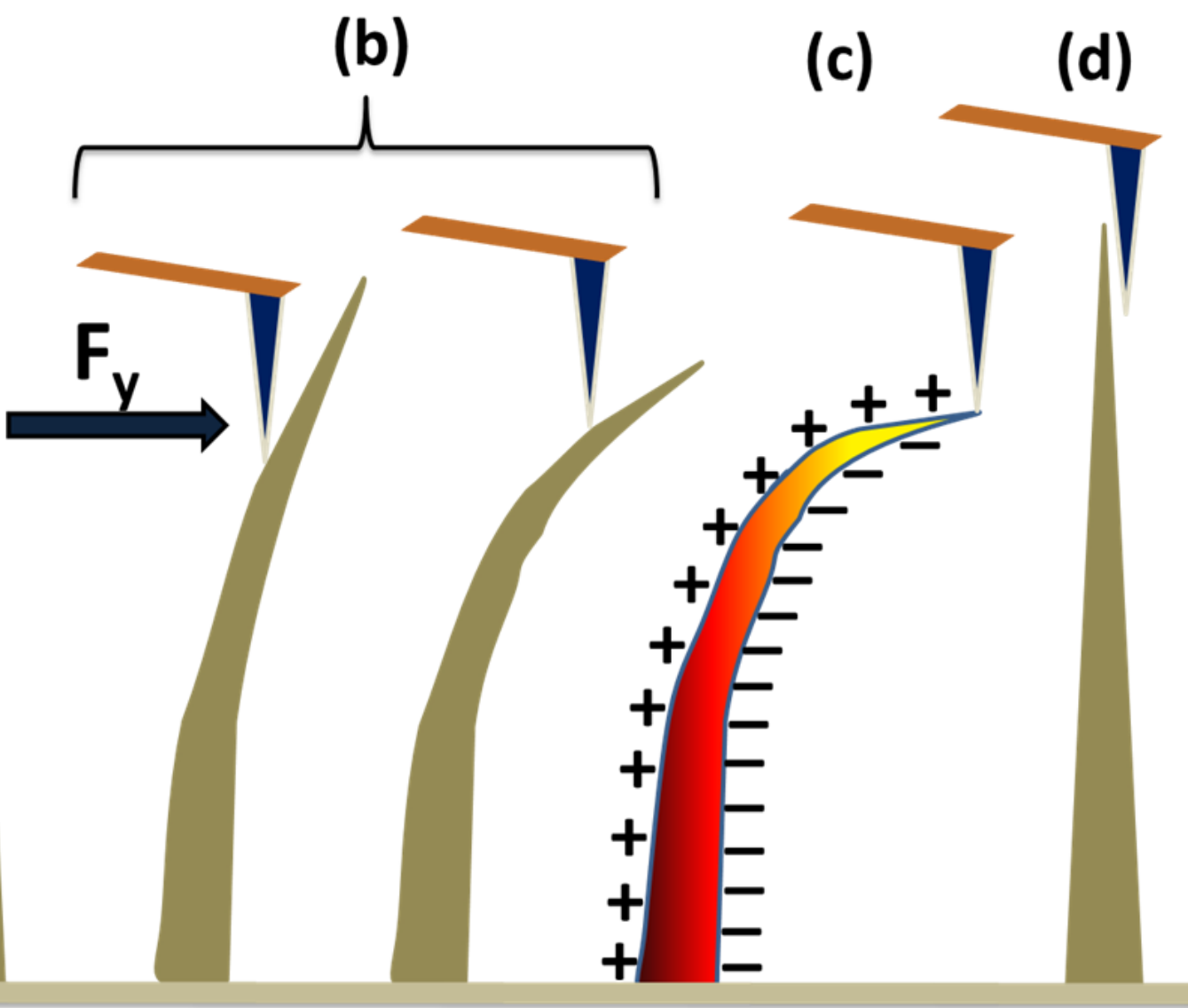

Figure 7: Schematic illustration of piezoelectricity generation mechanism from ZnO NNs, (a) AFM tip and NNs in relax form, (b) AFM tip and NNs in contact under the constant force, (c) positive and negative charges generating at maximum stretching/compression by tip, (d) NN after released from AFM tip. 
Figure 8:
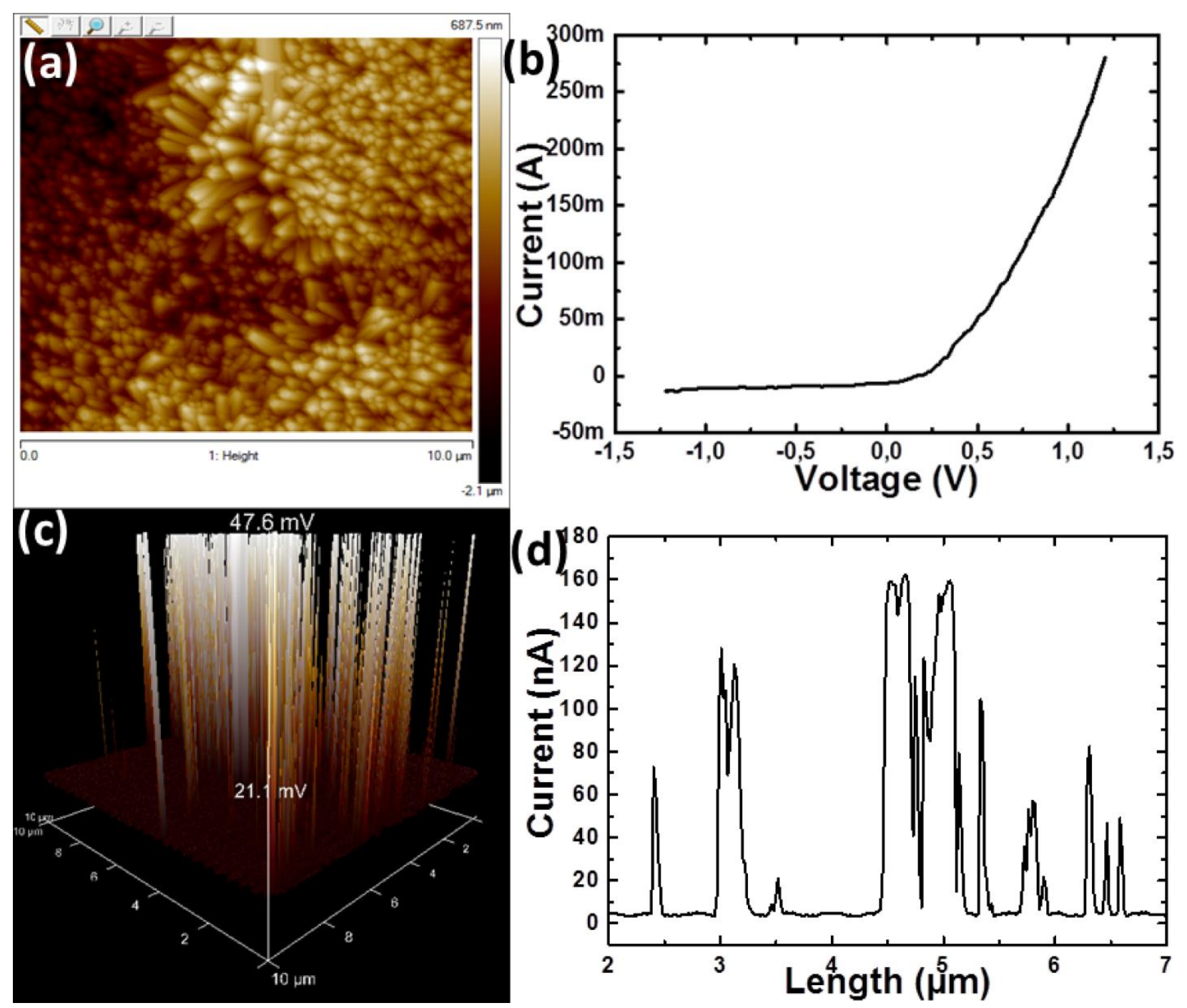

Figure 8: (a) Topographic images of $\mathrm{ZnO}$ NNs (b) Non-linear current voltage characteristics of $\mathrm{ZnO}$ NNs and AFM tip (c) Piezoelectric response in 3D (d) Line profile of the corresponding current outputs. 